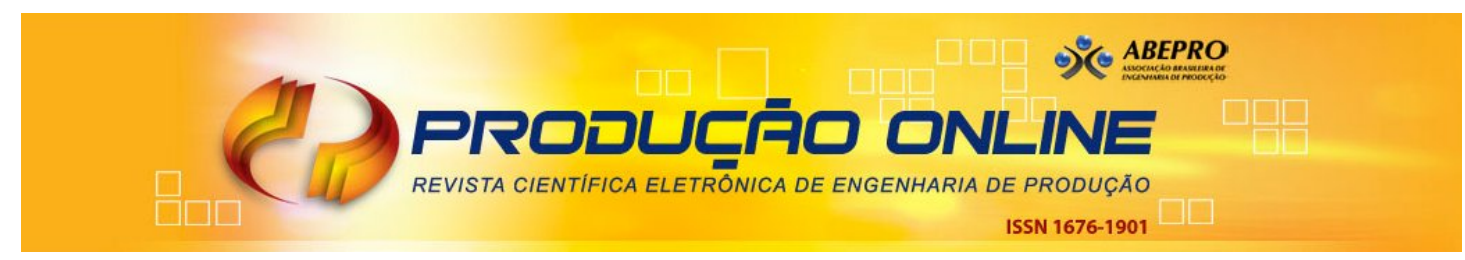

\title{
ATRIBUIÇÕES DE UM TIME DEDICADO À GESTÃO DA INOVAÇÃO EM UM GRUPO INDUSTRIAL BRASILEIRO: UM ESTUDO LONGITUDINAL BASEADO EM EVENTOS
}

\section{ASSIGNMENTS OF A DEDICATED TEAM FOR INNOVATION MANAGEMENT IN A BRAZILIAN INDUSTRIAL GROUP: A LONGITUDINAL STUDY BASED ON EVENTS}

\author{
Júlio Cézar Fonseca de Melo*E-mail: juliocfmelo@gmail.com \\ Raoni Barros Bagno* E-mail: rbagno@dep.ufmg.br \\ *Universidade Federal de Minas Gerais (UFMG), Belo Horizonte, MG
}

\begin{abstract}
Resumo: Inúmeros estudos têm ressaltado o desafio de empresas para gerir a inovação de forma sistemática. Vertente mais recente tem explorado casos em que essa prática está atrelada à constituição de uma função organizacional específica (i.e. Função Inovação - Fl). O conceito de função organizacional adotado nesse contexto é a existência de um grupo de pessoas identificável com uma missão definida na organização. Apesar de tal avanço, demanda-se maior entendimento acerca de como a Função Inovação se consolida ao longo do tempo no ambiente organizacional. O presente artigo traz um estudo da dinâmica de implantação dessa função em um grupo industrial brasileiro. A partir de levantamento longitudinal de 3,5 anos que mapeou a sequência de eventos envolvendo o time de trabalho da FI, buscou-se compreender como se deu o desdobramento de suas atribuições (e.g. geração de ideias, gestão de portfólio, busca por fomento e incentivos fiscais, formação de parcerias). Ao final, o estudo apresenta considerações sobre a dinâmica do processo de consolidação da Fl, sugere relações causais entre diferentes atribuições para posterior aprofundamento e identifica as atribuições mais relevantes para o caso analisado.
\end{abstract}

Palavras-chave: Gestão da inovação. Função organizacional. Função inovação.

Abstract: Numerous studies have highlighted companies' challenges to manage innovation systematically. Recent strands have explored cases where this practice is associated with a specific organizational function constitution (i.e. Innovation Function - IF). The concept of organizational function adopted in this context refers to an identifiable group with defined goals in the organization. Despite such progress, it is still necessary to understand how the Innovation Function consolidates over time in an organizational environment. In this regard, this paper presents the dynamics of implementation of the IF in a Brazilian industrial group. From 3.5 years longitudinal study focused on the sequence of events associated with the IF's team, the innovation function assignments emergence is discussed (e.g. idea generation, portfolio management, funding and tax incentives, partnerships). Based on these findings, we present considerations about the IF's consolidation process, suggest assignments causal relations and identify the most relevant assignments for the case.

Keywords: Innovation management. Organizational function. Innovation function.

\section{INTRODUÇÃO}

Mesmo empresas que apresentam bons exemplos de projetos de inovação, frequentemente os conduzem de forma infrequente, irregular e imprevisível 
O'CONNOR et al., 2008). Inovar de forma sistemática e regular é uma capacitação organizacional, cuja implantação incorreria em reconhecimento abrangente e aceitação da relevância da iniciativa para os objetivos de crescimento na organização (KELLEY et al., 2009). O'Connor et al. (2008) salientam que iniciativas como esta serão combatidas no ambiente interno das empresas, a menos que sejam protegidas e dotadas de validade, suporte e atenção da organização.

Neste contexto, uma possível alternativa para se promover a prática sistematizada da inovação nas empresas estaria na constituição de uma função organizacional específica para este fim (O'CONNOR et al., 2008). Na visão destes autores, uma função organizacional se define pela existência de um grupo de pessoas identificável e que possua a responsabilidade de conduzir e prestar contas acerca de uma missão específica da empresa. Bagno et al. (2017) alertam, entretanto, que a constituição de uma função organizacional para lidar com inovação não significa responsabilizá-la pelo desempenho total da empresa em inovação. Tal função teria o papel de catalisador ou facilitador da ocorrência de inovações, envolvendo as outras funções existentes. Assim, a Função Inovação (FI) não poderia ser considerada uma função de linha, mas de staff da organização.

Na visão de Wood Júnior (2004), uma transformação de natureza estrutural, institucional, estratégica, cultural, tecnológica, humana, ou de qualquer outro componente e que gere impacto em partes ou no conjunto da organização pode ser entendida como uma mudança organizacional. Assim, a constituição da FI em uma empresa pode ser entendida como elemento central de um processo de mudança. Kanter et al. (1992) enfatizam que a proporção em que uma mudança é ou não intencional, controlável e mensurável em prol de uma realidade futura desejada é algo a ser tratado pela gestão. Já Motta (1998) afirma que conduzir a inovação é algo mais complexo do que implícito na visão simplista e sequencial do planejamento-ação. Neste ponto, é importante ressaltar que o desempenho de uma empresa é resultante não somente da aplicação das iniciativas corretas, mas também da forma como são instauradas tais ações (KEUPP et al., 2012). Pode-se argumentar que os desafios à adoção da gestão da inovação de forma sistemática no ambiente industrial ainda são pouco explorados, sejam eles ligados aos aspectos internos da firma ou mesmo associados ao ambiente em que estão inseridas (GALIA; LEGROS, 2004). 
O'Connor et al. (2008) entendem que a construção de capacitação para inovar sistematicamente deva ocorrer em três fases: a "preparação do terreno", o início efetivo das atividades e a maturação, o que só ocorreria após um período de um ou dois anos. Em Bagno et al. (2017), propõe-se um conjunto de doze possíveis atribuições para a FI em uma organização (como, por exemplo, desenvolvimento de parcerias com universidades, gestão de portfólio de projetos de inovação ou engajamento com a captação de recursos financeiros para inovar). Tais atribuições correspondem ao desdobramento da missão central da FI em especialidades e tarefas específicas. Contudo, demanda-se maior esforço de investigação para se compreender como a FI é ou deve ser implantada ao longo do tempo e como suas atribuições emergem.

Diante do exposto, este artigo tem como objetivo principal o de descrever a dinâmica de implantação da FI em uma empresa de grande porte. Para isso, realizou-se uma análise longitudinal focada na sequência de eventos relacionados às ações desempenhadas pela FI e nos seus incrementos de recurso e estrutura. Tais eventos foram confrontados e relacionados com as atribuições identificadas na literatura para esta nova função. A partir deste ponto, foram tecidas considerações sobre o surgimento das atribuições da $\mathrm{FI}$ ao longo de seu processo de consolidação e também sobre sua legitimação no ambiente organizacional.

\section{REFERENCIAL TEÓRICO}

\subsection{Inovação: conceito, importância e regularidade}

O'Connor et al. (2008) colocam a falta de compreensão acerca do que seja a inovação como um problema gerador de confusões entre os times de trabalho. A questão estaria ainda relacionada a lentidão e ruína de diversos esforços para implantação de capacidade inovadora nas organizações. Na visão de Nelson e Winter (1982), praticamente qualquer mudança não trivial em produto ou processo, desde que não haja experiência anterior, poderia ser atestada como inovação. Conforme Bessant e Tidd (2009), a teoria sobre o processo de inovação teria sido construída, essencialmente, com base em inovações de cunho tecnológico, particularmente relacionadas ao setor industrial. O presente artigo adota o conceito 
proposto pelo Innovation Report de 2003, que define inovação como a exploração de novas ideias com sucesso. Isso envolveria, com frequência, novas tecnologias ou aplicações tecnológicas e sua importância estaria na geração de melhores produtos e serviços, processos de produção novos, mais eficientes e mais limpos e modelos de negócios superiores (DTI, 2003).

Vários autores delineiam também uma classificação de inovações em níveis de intensidade tais como básica, incremental intermediária, incremental avançada, arquitetural, radical (FIGUEIREDO, 2009), dentre outras. A utilidade em se discernir ou não a inovação em várias gradações distintas recairia no desafio da prática cotidiana das organizações em se moldar diferentes processos e tratativas de recursos para cada intensidade de inovação (BAGNO, 2014). Entretanto, é importante destacar que autores como Bessant et al. (2005) reconhecem que inovações radicais demandam fluxos e elementos organizacionais distintos em relação a inovações incrementais e demais atividades de rotina de uma empresa, perspectiva que reforça o papel de uma função organizacional direcionada à inovação.

Competências organizacionais e fatores de excelência operacional que elevaram organizações a grandes patamares de competitividade no passado não tem sido suficientes para garantir desempenho ou a própria sobrevivência da organização no futuro (TEECE, 2007; GIBSON, 2010). Na visão de Teece (2007), o sucesso organizacional estaria fortemente atrelado à descoberta e desenvolvimento de oportunidades, combinação de invenções geradas interna e externamente, transferência de tecnologia intra e entre empresas, proteção da propriedade intelectual, incremento das melhores práticas nos processos de negócio, tomadas de decisão imparciais e proteção contra a imitação e outras formas de replicação por parte dos rivais.

Entretanto, além de um conceito com fortes impactos para o desempenho organizacional, vertentes da literatura têm colocado a inovação como uma capacidade a ser construída. Tal capacidade estaria associada à fluidez e regularidade com a quais as oportunidades de inovação (ideias) seriam captadas e conduzidas através de um processo de inovação adotado pela empresa (c.f. Bagno et al., 2016), perpassando instâncias organizacionais, até se constituírem como inovações de fato. Isso se daria na venda ao mercado, difusão entre unidades 
organizacionais ou na implantação efetiva de novos processos organizacionais, conforme a natureza específica de cada oportunidade de inovação. Skarzynski e Gibson (2008) advogam por uma abordagem organizacional da inovação que a gerencie de maneira sistêmica e abrangente, criticando abordagens frequentes em determinadas organizações nas quais a inovação é resultado de brilhantismo individual e acaso.

Em Bagno et al. (2017), contudo, é relatado que as iniciativas empresariais de ponta registradas na literatura em torno da sistematização da inovação ainda são recentes, passam por mudanças constantes e não é possível identificar elementos que caracterizem objetivamente um estágio de maturidade. Maldonado et al. (2009) destacam a importância de uma abordagem conduzida pela alta direção (i.e. topdown) apoiada por ferramentas gerenciais (e.g. SWOT, Inteligência Competitiva, Desdobramento da Função Qualidade - QFD) para apoio à sistematização da atividade inovadora nas empresas.

\subsection{A Função Organizacional Inovação}

A definição de função organizacional em que se apoia este estudo é descrita em O'Connor (2012), que conceitua "função organizacional" como a existência de um grupo reconhecido e que possua a responsabilidade de conduzir e prestar contas acerca de uma missão específica da empresa. A experiência de pesquisa de O'Connor et al. (2008) aborda, no entanto, que o fenômeno da inovação como função organizacional ainda se encontra em estágio embrionário. Estes autores reforçam que a FI deva ser concebida de uma forma que não seja dependente de líderes de projeto especiais nem vulnerável ao perfil particular de líderes ou CEO's, condições momentâneas do mercado financeiro ou outros eventos que possam determinar a anulação dos esforços. Assim como outras funções organizacionais hoje estabelecidas, a inovação enquanto função organizacional demandaria, com o tempo, sua própria liderança, métricas, vocabulário, trajetórias de carreira, e poder permanente nas organizações.

Entre as responsabilidades da FI, O'Connor et al. (2008) identificam em seu estudo a construção e o norteamento de um portfólio de oportunidades de inovação, supervisionar a construção de capacidades de identificação de oportunidades, 
incubação (amadurecimento) e aceleração (desenvolvimento e difusão) destas oportunidades de inovação e a orquestração da relação com o restante da organização. Afirma-se ainda que tais relações tenderiam a mudar com frequência à medida em que a capacidade de inovação da empresa se desenvolvesse.

Em Bagno et al. (2017) é desenvolvido um estudo de caracterização da FI que se desdobra em seis perspectivas: (i) propósito geral da função inovação, (ii) origens dos grupos de inovação (eventos disparadores do processo de institucionalização), (iii) legitimidade e norteamento da função inovação no ambiente organizacional, (iv) envolvimento das pessoas, (v) atribuições da função inovação e (vi) perspectivas futuras da função inovação nos casos analisados. O trabalho se fundamentou em uma abordagem metodológica de estudos de casos múltiplos de 15 empresas industriais de grande porte no Brasil. Entre as perspectivas de análise deste estudo, as atribuições da FI se destacam por traduzir efetivamente as atividades desenvolvidas pela função (e pelas quais ela se torna identificável no ambiente organizacional). As atribuições (ATs) para a Fl identificadas são assim descritas:

- AT1 - Gestão de ideias internas: trata da captação de ideias de inovação de funcionários via programa de ideias, canais de empreendedorismo interno, workshops induzidos, etc.;

- AT2 - Parcerias com ICT's (Institutos de Ciência e Tecnologia): estabelecimento e gestão de parcerias com universidades ou centros de pesquisa;

- AT3 - Outras parcerias: estabelecimento e gestão de parcerias com associações empresariais, clientes corporativos, fornecedores, outras empresas, consultorias permanentes, governos, etc.;

- AT4 - P\&D (Pesquisa e Desenvolvimento): envolvimento direto da função inovação com atividades pesquisa, desenvolvimento tecnológico, questões técnicas dos projetos de inovação, etc. Como o P\&D pode justificar função específica na empresa e se relacionar com a função inovação, quando presente, de diversas formas, é reconhecido em Bagno (2014) que as atividades de P\&D possam não estar na alçada da Fl em diversos casos;

- AT5 - Portfólio e Gestão de Projetos de Inovação: Controle e gestão do portfólio de inovações em análise e desenvolvimento, planejamento 
individual dos projetos de inovação e envolvimento com seus aspectos gerenciais;

- AT6 - Propriedade Intelectual (PI): desenho das políticas de PI, gestão de patentes, mineração de patentes, estudos de formas de proteção, licenciamento, etc.;

- AT7 - Fomento e incentivos fiscais: busca de recursos externos, monitoramento de oportunidades de financiamento, elaboração de projetos para financiamento, etc.;

- AT8 - Cultura de Inovação: ações voltadas ao ambiente organizacional de inovação como cafés, eventos, iniciativas de comunicação, canais de envolvimento das pessoas em geral, etc.;

- AT9 - Desenvolvimento de Competências: iniciativas de capacitação de áreas ou pessoas em gestão de inovação ou voltadas à criação de competências tecnológicas para viabilizar projetos de inovação;

- AT10 - Gestão do Conhecimento (GC): ações como comunidades de prática; blogs para discussão de temas específicos relacionados à inovação; esforços de formalização e registro de experiências; registro e compartilhamento de lições aprendidas; ambientes para troca de conhecimento; etc., quando incluídos no escopo de inovação da empresa;

- AT11 - Prospecção (estratégica, tecnológica ou mercadológica): realização de predição de cenários futuros, seja de forma intuitiva ou por meio de ferramentas de mapeamento;

- AT12 - Novos negócios e investimentos de risco: atividades voltadas à criação de novos negócios, aplicações de tecnologias/produtos atuais em mercados ainda não explorados; formação de spin-offs, atuação como investidor de capital de risco, etc.

Argumenta-se ainda em Bagno et al. (2017), que a constituição da $\mathrm{FI}$ não requer igualdade de importância entre as atribuições exercidas, nem tampouco exige-se que todas as atribuições sejam exercidas para se configurar a existência ou não da FI. Já quanto à legitimidade e estruturação da FI nas empresas, além de um grupo de pessoas central, identificável por responder diretamente pela missão da função, três outras instâncias são identificadas no estudo de Bagno et al. (2017): comitês estratégicos, pontos focais e times de projeto (Figura 1). Tais instâncias se 
ligariam então ao grupo central para direcionar o trabalho deste grupo, catalisar ligações internas ou mesmo assumir responsabilidades complementares. O(s) comitê(s) estratégico(s) são norteadores da FI quanto ao alinhamento de seus objetivos e atividades com a estratégia da organização, além de concentrarem decisões sobre alocação de recursos e remoção de barreiras organizacionais em prol da Fl. Os chamados pontos focais constituem-se de pessoas formalmente alocadas em outras funções da empresa (e.g. Financeiro, Recursos Humanos, Engenharia), mas que atuam como extensões da FI de tempo parcial para realização de atividades pertinentes às vocações da função local. Finalmente, os times de projeto são estruturas temporárias que trabalham diretamente nos projetos de inovação e podem envolver pessoas formalmente alocadas em várias áreas da organização, conforme as demandas de competências particulares a cada projeto.

Figura 1 - Instâncias organizacionais da função inovação ligadas ao grupo central de inovação



Fonte: adaptado de Bagno et al. (2017)

\section{METODOLOGIA}

A estratégia metodológica utilizada é o Estudo de Caso (YIN, 2004), por meio de abordagem longitudinal e retrospectiva (EISENHARDT; GRAEBNER, 2007). A pesquisa foi conduzida em um grupo industrial brasileiro (Grupo $\Phi$ ) sendo o período de coleta dos dados outubro de 2010 - dezembro de 2013. Um dos autores deste 
trabalho (Pesquisador 1) participou ativamente do processo de consolidação da FI no Grupo $\Phi$, atuando como gestor de inovação no período em questão. Esta responsabilidade lhe foi atribuída no início do processo por um dos acionistas (e vice-presidente) do Grupo. Dados secundários foram coletados de documentos gerados no horizonte de tempo especificado (atas de reunião, apresentações e relatórios gerenciais, gravações, e-mails, notícias oficiais).

Inicialmente, procurou-se levantar eventos associados à gestão da inovação (EVs), tidos como relevantes. A pesquisa caracteriza-se, portanto, como processual e eventos constituem os dados básicos por meio dos quais se buscou explicar como a implementação da FI ocorreu (LANGLEY, 1999). Os eventos foram agrupados segundo duas variáveis: (i) empresa do Grupo $\Phi$ a que se relaciona o evento, e (ii) semestre de ocorrência. Criou-se ainda uma classificação "outros", para eventos que não estivessem associados a uma empresa específica (eventos de impacto comum a todo o Grupo $\Phi$ ).

Posteriormente, foi feita uma análise da granularidade dos eventos obtidos. A divisão por semestre propiciou, em alguns momentos, o apontamento de eventos consideravelmente abrangentes, que poderiam ser divididos em outros mais específicos. Uma nova análise documental foi então realizada, tanto para revisar a lista de eventos e relacioná-los a um horizonte de tempo mensal, como também para associar a cada um deles, evidência documental que justificasse sua ocorrência.

Em um terceiro momento, uma narrativa do caso foi elaborada, a partir da lista de eventos. Esta medida foi adotada por ser uma estratégia reconhecida para descrição de uma história (LANGLEY, 1999). Ademais, precisava-se de um instrumento capaz de apresentar com riqueza de detalhes o caso ao outro pesquisador (Pesquisador 2), para que pudesse apoiar na análise dos dados. Outro benefício advindo dessa ação foi que, ao descrever o caso, o Pesquisador 1 recordou-se de outros fatos relevantes, adicionando 6 (seis) novos eventos à lista anterior, totalizando 129 eventos. O quadro (Quadro 3) contendo os eventos identificados pelo Pesquisador-Autor para o período está disponível no ADENDO I.

Assim, iniciou-se a análise dos dados a partir da elaboração de uma matriz. No eixo vertical foram listados os eventos, e no horizontal, as 12 (doze) atribuições (ATs) da FI definidas por Bagno (2014). O Pesquisador 1 estabeleceu a correlação entre eventos e as atribuições, marcando um " $X$ " nos cruzamentos em que a relação 
era válida. A título de exemplo, ao evento 64 - Ideia do P\&D-22 - foi correlacionado à atribuição AT1 - "Gestão de ideias internas", enquanto ao evento 6 - Início da interação com setor de auditoria de projetos de inovação da CEMIG, à AT3 - "Outras parcerias". Neste momento, dois pontos de atenção emergiram: (i) existiam eventos que poderiam ser associados a mais de uma atribuição, porém, com diferentes níveis de aderência; (ii) determinados eventos não se enquadravam em nenhuma das atribuições de forma direta. Em relação a (i), o evento 23 - Parceria com Centro Federal de Educação Tecnológica de Minas Gerais (CEFET-MG) e microempresa para submissão do P\&D-7 em chamada do "EDITAL CEMIG-FAPEMIG" (uma parceria entre a Companhia Energética de Minas Gerais - CEMIG e a Fundação de Amparo à Pesquisa do Estado de Minas Gerais - FAPEMIG), por exemplo, relacionava-se as atribuições AT2, AT3 e AT7, simultaneamente. Em relação ao item (ii), o evento 1 - Contratação do Pesquisador-Autor para assumir o "Núcleo de Gestão da Inovação" - pode ser tomado como um evento relacionado à estruturação da FI, munindo-a de recursos e formalizando suas instâncias organizacionais (Figura 1) e não a uma de suas atribuições. Este tipo de evento, portanto, não foi associado a nenhuma das atribuições naquele instante.

A matriz preenchida preliminarmente foi então validada junto ao outro autor deste estudo. Foram discutidas as correlações de todos os eventos e estabelecidas as seguintes ações para contornar os desafios pontuados anteriormente: (i) criou-se uma escala de pontuação com notas "1" e "2" para correlacionar eventos e atribuições, sendo "1" para relações tidas como fracas e/ou indiretas e "2" para aquelas de caráter forte e/ou direto; (ii) foi criada uma nova categoria para estrutura. O evento 30 - Parceria com Instituto Euvaldo Lodi (IEL) no "PROGRAMA DE GERAÇÃO DE IDEIAS" - ilustra bem o ponto (i). Foi associada nota "2" para AT1, pela relação direta, e "1" para AT3 pois o Grupo $\Phi$ já estabelecera no passado outras parcerias com o IEL, mas o este novo evento promovia o fortalecimento da relação entre essas organizações.

O objetivo do preenchimento da matriz, além da classificação de cada evento, foi prover uma base para se analisar o nível de atividade de cada atribuição da função inovação ao longo do tempo, no caso estudado. Para isso, procedeu-se com uma contagem simples do número de ocorrências de eventos por atributo e por mês. A partir disso, elaborou-se curvas com nível de atividades nas atribuições da função 
inovação ao longo do tempo (ver Figura 2 e Figura 3 no tópico 5) a partir de técnica de Médias Móveis Ponderadas (MPP) sobre a contagem simples previamente feita,

Essa abordagem foi escolhida pela possibilidade de incorporar o caráter residual do impacto de eventos ao longo do tempo e suavizar as curvas de "nível de atividade das atribuições". O horizonte de tempo considerado para o cálculo das médias móveis foi de 6 (seis) meses e os pesos utilizados variaram de 1 a 6 (peso 1 para eventos que aconteceram há 6 meses em relação ao período em análise, 2 para eventos ocorridos há 5 meses, e assim por diante). A utilização deste intervalo no cálculo das médias móveis não considerou de forma completa os eventos ocorridos nos primeiros 6 (seis) meses do horizonte de tempo (outubro de 2010 a fevereiro de 2011). Entretanto, esta limitação é parcialmente mitigada por existirem poucos eventos neste período.

\section{O CASO}

O Grupo $\Phi$ atua em diversos nichos de mercado e gera mais de 3.000 empregos diretos. Sua atividade principal é o fornecimento de soluções em energia e automação, sendo formado em sua atividade produtiva, na época do estudo, por 8 (oito) empresas (ver Quadro 1). O problema abordado neste estudo está associado à implementação da FI neste contexto.

Quadro 1 - Empresas do Grupo $\Phi$

\begin{tabular}{|c|c|}
\hline$\#$ & DESCRIÇÃo \\
\hline EP1 & $\begin{array}{r}\text { Soluções Turn-Key em sistemas de energia e automação, e equipamentos elétricos e } \\
\text { eletromecânicos. }\end{array}$ \\
\hline EP2 & $\begin{array}{r}\text { Fabricante de transformadores de corrente, transformadores potencial e conjuntos de } \\
\text { medição. }\end{array}$ \\
\hline EP3 & Projeto e fabricação de transformadores de potência. \\
\hline EP4 & $\begin{array}{r}\text { Soluções completas EPC (Engineering, Procurement and Construction) de projeto, montagem } \\
\text { eletromecânica, obras civis, comissionamento, start-up e operação assistida. }\end{array}$ \\
\hline EP5 & Soluções integradas em engenharia multidisciplinar. \\
\hline EP6 & Tecnologias de ponta destinadas ao mercado de Petróleo \& Gás. \\
\hline EP7 & $\begin{array}{r}\text { Desenvolvimento de oportunidades na área de geração e transmissão de energia elétrica, tais } \\
\text { como PCHs (Pequenas Centrais Hidrelétricas) e outras fontes renováveis. }\end{array}$ \\
\hline EP8 & Exploração e produção de óleo e gás. \\
\hline
\end{tabular}

Fonte: elaborado pelos autores 
No início do caso, o Pesquisador-Autor deparou-se com dicotomias acerca da percepção das atividades de inovação no Grupo $\Phi$. A alta direção defendia que o Grupo $\Phi$ teria sobrevivido ao longo de seus mais de 35 anos de história por meio do desenvolvimento bem-sucedido de inovações tecnológicas. Gerências e grupos intermediários afirmavam que as empresas não eram inovadoras ("são apenas montadores"; "não tem tecnologia alguma") e nem tampouco tinham interesse em investir em inovação. Outra contradição residia nos discursos dos dois grupos: o primeiro declarava que queria investir em inovações mas faltavam boas ideias; o segundo alegava ter ideias, mas que não haviam oportunidades de colocar seus anseios perante a alta direção e também, que não seriam valorizados se o fizessem.

No ano de 2008 houve uma iniciativa que pode ser interpretada como uma tentativa de implantação de FI no Grupo Ф. Foi criado um setor chamado "NGI" (Núcleo de Gestão da Inovação). Esse departamento era subordinado à diretoria da EP1. Um dos autores (Pesquisador-Autor), no início do caso (outubro de 2010), fora contratado para assumir a gestão dessa instância (i.e. Orquestrador da FI). Até aquele momento, não se podia afirmar que existia uma função organizacional inovação consolidada. A EP1, maior empresa do Grupo, acabara de encerrar um dos projetos de desenvolvimento de um equipamento de testes específico para sua linha de montagem com investimento próprio. No entanto, não se sabia o montante financeiro total investido no projeto ou o retorno que o mesmo traria para a empresa. A EP2 era uma start-up voltada ao desenvolvimento tecnológico, tendo desenvolvido alguns protótipos em conjunto com um Centro de Pesquisa de uma companhia de exploração de petróleo brasileira. No entanto, era rotulada no Grupo $\Phi$ como uma empresa que apenas gerava despesas.

O entendimento de que a inovação, enquanto função organizacional, foi consolidando-se durante o período em questão perfazendo uma trajetória evolutiva (o que valida o caso para ser analisado neste estudo) é fundamentada nos seguintes marcos, presentes no caso ao final do período de análise:

- Criação de um grupo de pessoas bem identificado com a missão de captar e acelerar oportunidades de inovação (colaboradores diretamente vinculados ao NGI, outros indiretos vinculados a um Comitê de Inovação, e agentes diversos participando sistematicamente de atividades relacionadas à temática); 
- Coleta e avaliação de aproximadamente 50 ideias de novos produtos e tecnologias;

- Gestão de 13 (treze) projetos de PD\&l (Pesquisa, Desenvolvimento e Inovação);

- Formação de parcerias formais (i.e. acordos jurídicos) com agências de fomento do governo federal, bancos de investimento públicos e privados, fornecedores, clientes, universidades, agências de apoio à inovação, empresas de consultoria, dentre outros;

- Aprovação de montante de recursos expressivos (a sétima parte do faturamento anual do grupo) para apoio a projetos, englobando incentivos fiscais, financiamentos reembolsáveis e não reembolsáveis;

- Promoção de pelo menos um projeto de inovação com cada empresa do Grupo $\Phi$;

- Suporte à negociação bem sucedida de investimento direto (i.e. equity) na EP2;

- Reconhecimento subjetivo do valor NGI para o Grupo $\Phi$, tanto por agentes internos quanto externos.

\section{RESULTADOS: ANÁLISE E DISCUSSÕES}

As análises destacadas no tópico de Metodologia proporcionaram a elaboração das Figuras 2 e Figura 3. Estas mostram a intensidade do esforço relacionado a cada atribuição da $\mathrm{Fl}$ ao longo do tempo para o caso estudado. Os valores do eixo vertical de cada gráfico foram dados pelo cálculo das médias das médias móveis mensais do número de eventos ligados a cada atribuição segundo seu peso. Dividiu-se o conjunto em dois grupos: um grupo formado pelas atribuições que tiveram maior volume de eventos a elas associados (estas foram chamadas então de atribuições principais para o caso em questão); outro grupo formado pelas demais atribuições (chamadas de secundárias). As últimas, além do menor volume de atividades ao longo do período, são caracterizadas, em sua maioria, pelo surgimento posterior às consideradas principais no processo avaliado. O ponto de corte utilizado para separar as atribuições consideradas principais daquelas 
secundárias foi a média das médias móveis ponderadas mensais de todas as atribuições (ver resultados no Quadro 2). 


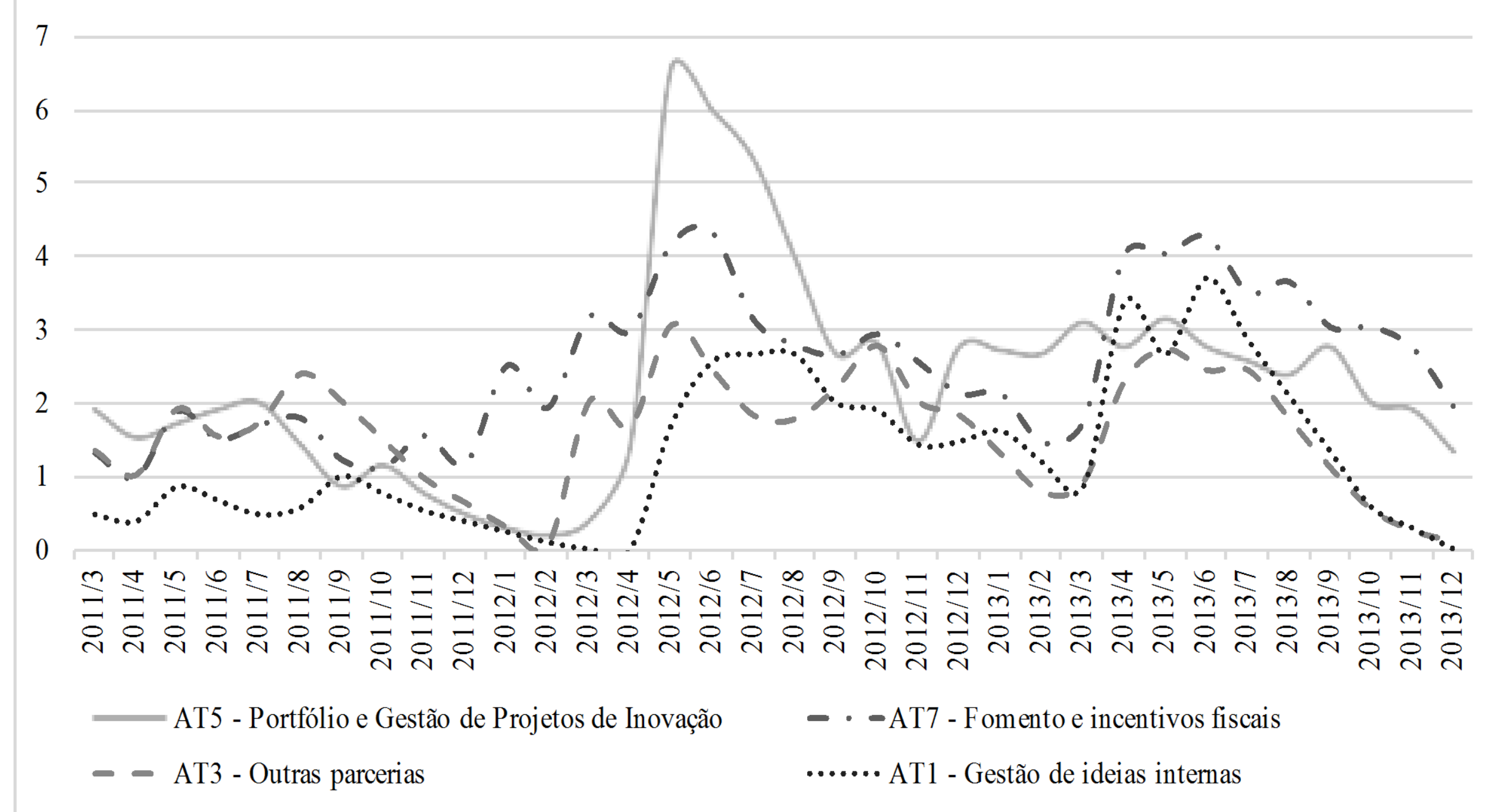






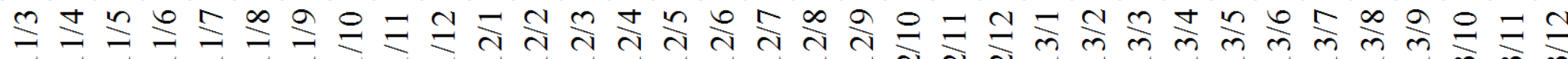

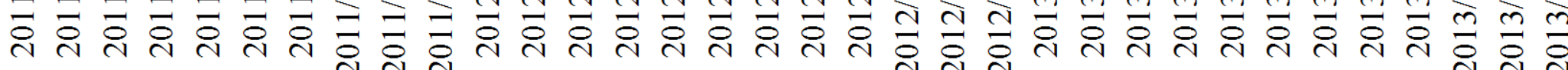
AT2 - Parcerias com ICT

- AT9 - Desenvolvimento de Competências

....... AT12 - Novos negócios e investimentos de risco

- AT8 - Cultura de Inovação

$\bullet \cdot-A T 6$ - Propriedade Intelectual (PI)

AT4 - P\&D (Pesquisa e Desenvolvimento)

AT10 - Gestão do Conhecimento (GC)

AT11 - Prospecção (estratégica, tecnológica ou mercadológica) 
Quadro 2 - Ranking das atribuições conforme sua relevância para consolidação da função inovação no Grupo $\Phi$

\begin{tabular}{|c|c|c|}
\hline$\#$ & ATRIBUIÇÃO & $\begin{array}{c}\text { MÉDIA DAS MÉDIAS } \\
\text { MÓVEIS PONDERADAS } \\
\text { MENSAIS }\end{array}$ \\
\hline 1 & AT7 - Fomento e incentivos fiscais & 2,52 \\
\hline 2 & AT5 - Portfólio e Gestão de Projetos de Inovação & 2,28 \\
\hline 3 & AT3 - Outras parcerias & 1,57 \\
\hline 4 & AT1 - Gestão de ideias internas & 1,28 \\
\hline 5 & AT2 - Parcerias com ICT's & 0,77 \\
\hline 6 & AT9 - Desenvolvimento de Competências & 0,36 \\
\hline 7 & AT12 - Novos negócios e investimentos de risco & 0,25 \\
\hline 8 & AT8 - Cultura de Inovação & 0,14 \\
\hline 9 & AT6 - Propriedade Intelectual (PI) & 0,12 \\
\hline 10 & AT4 - P\&D (Pesquisa e Desenvolvimento) & 0,10 \\
\hline 11 & AT10 - Gestão do Conhecimento (GC) & 0,06 \\
\hline \multirow[t]{2}{*}{12} & AT11 - Prospecção (estratégica, tecnológica ou mercadológica) & 0,06 \\
\hline & MÉDIA DAS MÉDIAS & $\overline{0,79}$ \\
\hline
\end{tabular}

Nota-se que, em maior ou menor grau, todas as atribuições propostas por Bagno (2014) foram identificadas no caso analisado, apesar de não ser esta uma premissa básica para a FI. A análise dos dados também não revelou nenhum evento que não apresentasse aderência a pelo uma das atribuições listadas, à exceção daqueles que foram categorizados como eventos de estruturação da FI.

A partir de análise do contexto do caso paralelamente à Figura 2 e à Figura 3 , chega-se a 4 (quatro) implicações gerais inerentes ao processo de consolidação da função organizacional inovação, como detalhado nos tópicos abaixo.

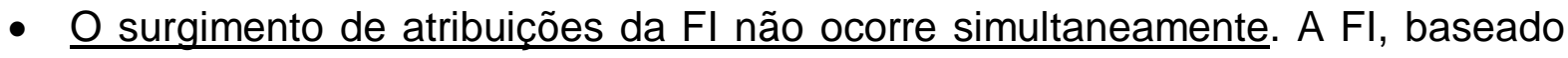
no caso tratado, nasce com algumas atribuições incipientes e, ao longo de seu processo de consolidação, estas atribuições tendem a ganhar regularidade (tornando-se rotinas) e novas atribuições podem emergir. É importante notar que uma nova função organizacional não somente compete por recursos junto a outras funções já consolidadas como também sofre da falta de resultados anteriores que justifiquem tal alocação de recurso. No caso da FI, sendo uma função portadora de futuro pela natureza de sua missão, a justificativa de recursos baseada em resultados é ainda mais desafiadora, o que faz com que sua institucionalização ocorra de forma ainda mais gradual.

- O esforço em torno de cada atribuição varia ao longo do período de consolidação da FI. No caso analisado, limitações de recursos (principalmente humanos) provocavam uma constante reavaliação de prioridades. Observou-se assim uma concentração de esforços da FI em tipos específicos de atividades em diferentes momentos. Como exemplo, a existência de montante significativo de projetos em andamento pode reduzir o foco em captação de novas ideias:

- As atribuições se inter-relacionam e tais inter-relações não somente se intensificam na medida em que a FI se consolida no ambiente organizacional como catalisam o surgimento de novas atribuições. A demanda por esforço em torno das atribuições principais e 0 desdobramento das atividades frequentemente disparava novas atribuições. A captação de uma ideia ou projeto, por exemplo, demandava como consequência direta esforços na gestão 
de portfólio e na gestão de projetos. Gargalos na gestão de projetos evidenciavam a necessidade de suporte em gestão de competências ou gestão de conhecimento. Outro exemplo é que projetos executados junto a agentes de fomento externo ou envolvendo parceiros suportavam a definição de práticas específicas de gestão de projetos de P,D\&I. Um bom exemplo é a elaboração e revisão de cronogramas e entregas de relatórios exigidos por entidades apoiadoras e que acabavam sendo generalizadas para outros projetos na empresa.

- A consolidação da Fl é um fenômeno gradual e que se dá no médio-longo prazo. Não se entende no contexto deste trabalho a consolidação de uma função organizacional como uma variável binária, de forma que se possa definir um ponto no tempo que separa a não consolidação da consolidação. Entretanto, ao se acompanhar o caso longitudinalmente, as diferenças de status da função no início e fim do período de análise se tornam notórias. No momento inicial, há maior vulnerabilidade e inconstância do time envolvido, baixo volume de atividades, poucas atribuições identificáveis, pouco reconhecimento no ambiente organizacional e poucos recursos alocados (físicos, humanos e financeiros). Um ganho perceptível de legitimidade nestes aspectos ocorre em um período da ordem de 2 (dois) anos. Esse aspecto é fundamental para nortear expectativas e cobrança por resultados do trabalho em organizações inseridas em desafios similares e converge com as observações feitas por O'Connor et al. (2008).

Algumas implicações reveladas pela análise dos dados foram consideradas específicas do caso estudado, mas emergem como referências de análise para novos casos em busca de generalização, como mostrado a seguir:

- Quatro atribuições mostraram ser de maior importância para a consolidação da Fl. São elas: AT7 - "Fomento e incentivos fiscais"; AT5 - "Portfólio e Gestão de Projetos de Inovação"; AT3 - "Outras parcerias"; AT1 - "Gestão de ideias internas". Convergindo com a visão dada em Bagno (2014), fomento e incentivos fiscais se mostraram como fortes disparadores da formação da $\mathrm{Fl}$, o que é moldado, em grande parte, pelo contexto das políticas locais de C\&T. Já as atividades inerentes a portfólio e gestão de projetos de inovação podem ser interpretadas como foco central da FI na missão de catalisar a ocorrência de inovações. No entanto, o destaque para as atribuições de gestão de ideias internas e outras parcerias são específicos do caso, não convergindo com a ordenação encontrada nas empresas pesquisadas por Bagno (2014) nas quais a gestão de ideias era uma das atribuições de menor intensidade e as parcerias com outras empresas eram significativamente menos enfatizadas do que com ICT'S.

- A busca por Fomentos e Incentivos Fiscais (AT7) é um legitimador de curto prazo da FI no nível estratégico da organização. Foi notória no caso analisado a influência destas atividades na percepção da FI pela alta gerência devido ao montante de recursos financeiros captados. Tal situação pode ser exemplificada pelo evento 32 - Interação do NGI com Planejamento Estratégico. Ademais, na 
reunião que marca o início do caso, houve menção direta pelo então vicepresidente do grupo para direcionamento de esforços nesse sentido. Esta diretriz foi reforçada, em março de 2011, quando o então gerente de produtos declara que o reconhecimento para a função aconteceria condicionado à captação de recursos financeiros externos (evento 14 - Reunião de alinhamento do P\&D-5 com setor de "Engenharia do Produto"). Maldonado et al. (2014) destacam que as empresas deveriam preparar estruturas organizacionais e cultura para captar recursos advindos de agência de fomento. Neste estudo, evidencia-se um caminho inverso - recursos financeiros de fontes externas foram utilizados como o principal mecanismo para a estruturação de um sistema de gestão da inovação.

- O início da implantação da Fl é marcado por conflitos com outras funções, mas estes são paulatinamente substituídos por relações de colaboração na medida em que o perímetro de atuação torna-se mais claro. O uso de pontos focais em outras funções da organização não somente suaviza a demanda de recurso próprio da FI no início de suas atividades como acelera a formação de processos de comunicação horizontal com tais funções. Situações como a descrita pelo evento 17 - Problemas com movimentação de contas bancárias de projetos junto ao Departamento Financeiro - aconteceram no início do caso, mas não foram observadas posteriormente. Ademais, com a prerrogativa da AT7, percebe-se um movimento de ampliação, já no segundo semestre do ano de 2011, do envolvimento da FI com outras empresas do Grupo Ф (evento 29 Visita à EP-2 e identificação do P\&D-9).

- Aspectos exógenos à firma são fortes moldadores da FI. Alguns instrumentos de apoio à inovação são específicos para segmentos de atuação do Grupo $\Phi$ (como o Programa de P\&D da ANEEL - Agência Nacional de Energia Elétrica) ou para o setor industrial (Editais/iniciativas do Serviço Nacional de Aprendizagem Industrial - SENAI e Serviço Social da Indústria - SESI). Essas iniciativas pressupõem envolvimento com agentes (parceiros) específicos como précondição para apresentação de projetos de P,D\&l (com concessionárias de energia, no primeiro caso, e unidades do sistema SESI/SENAI, no segundo). Outros contatos com o meio externo miravam no acesso a competências distintivas, com objetivo de uso pontual ou internalização pela FI e podem ser considerados, portanto, questões ligadas à infraestrutura local de C,T\&l. Como exemplo, tomemos o evento 30 - Parceria com IEL no "PROGRAMA DE GERAÇÃO DE IDEIAS". Destaca-se ainda a cooperação com empresas privadas que podem ser consideradas concorrentes do Grupo $\Phi$ em outros segmentos como no evento 23 - Parceria com CEFET-MG e microempresa para submissão do P\&D-7 em chamada do "EDITAL CEMIG-FAPEMIG".

- o gradativo reconhecimento da FI no ambiente organizacional desembaraça a captação de recursos humanos para a função, assim como o envolvimento de colaboradores em outras funções. A adesão de colaboradores internos do Grupo $\Phi$ à $\mathrm{FI}$ era baixa no início do período de análise, o que era evidenciado, por exemplo, pelo fraco desempenho na geração de ideias. Não havia perspectiva 
de crescimento ou promoção das pessoas pelo envolvimento nas iniciativas de inovação. Além disso, os agentes internos imaginavam que, ao assumir atividades fora de seu escopo básico na organização, estariam automaticamente aumentando sua carga de trabalho. No entanto, ao longo do período de análise, o reconhecimento gradativo da FI pelo Conselho de Administração, assim como a criação de um Comitê de P,D\&I ou o reconhecido papel da FI no atendimento a demandas externas como os Programas da ANEEL, foram elementos que se destacaram por atrair o interesse das pessoas em se envolver com a nova função.

\section{CONCLUSÕES}

A implementação de uma gestão sistemática da inovação é tarefa de fundamental importância para sobrevivência das empresas no ambiente industrial contemporâneo (Keupp et al., 2012). Bagno et al. (2017) mostram a criação de uma nova função organizacional específica como uma alternativa para se chegar a este fim. Neste contexto, o presente trabalho buscou levantar evidências empíricas acerca de como se consolida a função inovação em uma empresa específica, sendo ela elemento central de um processo de mudança organizacional. O caso analisado evidenciou não somente o surgimento paulatino das atribuições ligadas à $\mathrm{FI}$ como também suas inter-relações, relações com outras funções e também com o ambiente externo à organização.

Como limitações do estudo, é importante reconhecer as questões intrínsecas ao estudo de caso único como limitador da generalização das análises (YIN, 2004). Outro ponto é que a construção da lista de eventos considerados relevantes foi executada apenas por um participante. A validação da mesma por outros atores do caso deve ser considerada na evolução deste estudo. Outro ponto de atenção está na utilização da média móvel para avaliação efeitos residuais dos eventos ao longo dos períodos subsequentes. Os pesos atribuídos não necessariamente representam fielmente o impacto dos eventos no horizonte de tempo futuro. Uma abordagem metodológica alternativa (i.e. mapas visuais) para avaliação do problema abordado neste trabalho é detalhada em Melo et al. (2016).

Do ponto de vista da prática das organizações, a dinâmica de consolidação da FI no caso relatado pode fornecer importantes insights para abordagens planejadas do processo de implantação da função em outras empresas. Neste contexto, o presente estudo apresenta de forma detalhada a trajetória de um caso de implantação da FI e sugere pontos de partida. O caso evidencia também a importância do alcance de alguns marcos intermediários como elementos de suporte ao processo de implantação, como é o caso da captação de recursos externos, realização de parcerias formais e aceleração das iniciativas de ideação.

Como principal contribuição para a teoria de gestão da inovação, o estudo aprofunda na compreensão da natureza da FI nas organizações e sobre suas formas de implantação e proteção no ambiente organizacional. O estudo evidencia também o impacto de políticas públicas e da infraestrutura de C,T\&l na gestão interna da organização no processo de implantação da FI. 
Entre as perspectivas de continuidade deste estudo estão o aprofundamento no próprio caso a partir de um período de análise mais extenso e com o envolvimento de um maior número de informantes. Outra oportunidade está na extensão deste modelo de análise para outras empresas e setores que vem investindo na FI como forma de institucionalizar atividades regulares de gestão da inovação. Sugestões para pesquisas futuras podem ainda envolver: o uso de novas abordagens de modelagem da cadeia de eventos, buscando identificar relações de causalidade entre eles; identificação de eventos críticos para a consolidação da Fl; avaliação da relação entre estratégia e estrutura na formação da FI (c.f. CHANDLER JR, 1998); avaliação do processo de consolidação da função organizacional inovação sob outras perspectivas teóricas, como pelos tipos de processos de formação estratégica (c.f. MINTZBERG et al., 2005).

\section{REFERÊNCIAS}

BAGNO, R.B. Inovação como uma nova função organizacional: caracterização a partir da experiência de empresas industriais de grande porte no Brasil. 2014. 199p. (Doutorado). Escola Politécnica, USP, São Paulo.

BAGNO, R.B.; SALERNO, M.S.; DA SILVA, D.O. Models with graphical representation for innovation management: a literature review. R\&D Management, 2016. ISSN 1467-9310.

BAGNO, R.B.; SALERNO, M.S.; DIAS, A.V.C. Innovation as a new organizational function: evidence and characterization from larg

BESSANT, J. et al. Managi e industrial companies in Brazil. Production Journal, 2017. ng innovation beyond the steady state. Technovation, v. 25, n. 12, p. 1366-1376, 2005. https://doi.org/10.1016/j.technovation.2005.04.007

BESSANT, J.; TIDD, J. Inovação e Empreendedorismo. Porto Alegre: Bookman, 2009. $512 \mathrm{p}$.

CHANDLER JR., A. D. (1998). Ensaios para uma teoria histórica da grande empresa. Rio de Janeiro: FGV, 1998.

DTI. Innovation Report: Competing in the global economy: the innovation challenge. DTI. 2003

FIGUEIREDO, P.N. Gestão da Inovação: conceitos, métricas e experiências de empresas no Brasil. Rio de Janeiro: LTC, 2009.

GALIA, F.; LEGROS, D. Complementarities between obstacles to innovation: evidence from France. Research Policy, v. 33, n. 8, p. 1185-1199, 2004. https://doi.org/10.1016/j.respol.2004.06.004

GIBSON, R. Making Innovation a Systemic Capability. 2010 Disponível em: < http://www.rowangibson.com/images/stories/RGres/RGArticles/A116_MakingInnovationASys temicCapability_IdeaConnection_USA_Apr2010.pdf >.Acesso em: 11 Jan 2013.

KANTER, R.M.; STEIN, B.A.; JICK, T.D. The Challenge of Organizational Change: How companies experience it and leaders guide it. New York: Free Press, 1992.

KELLEY, D.J.; PETERS, L.; O'CONNOR, G.C. Intra-organizational networking for innovation-based corporate entrepreneurship. Journal of Business Venturing, v. 24, n. 3 , p. 221-235, 2009. https://doi.org/10.1016/j.jbusvent.2008.05.010

KEUPP, M.M.; PALMIE, M.; GASSMANN, O. The Strategic Management of Innovation: A Systematic Review and Paths for Future Research. International Journal of Management Reviews, v. 14, n. 4, p. 367-390, 2012. https://doi.org/10.1111/j.1468-2370.2011.00321.x

LANGLEY, A. Strategies for theorizing from process data. Academy of Management Review, v. 24, n. 4, p. 691-710, 1999. 
MALDONADO, M.U.; DIAS, N.; VARVAKIS, G. Managing innovation in small hightechnology firms: a case study in Brazil. Journal of Technology Management \& Innovation, v. 4, n. 2, p. 130-142, 2009. ISSN 0718-2724.

MALDONADO, M.U.; VAZ, C.R.; DIAS, N. Innovation Management in Brazilian Smes: Mapping out Innovation Practices and Performance. IAMOT (International Association for Management of Technology) 2014.

MELO, J.C.F.D. et al. Building innovation capability: lessons from a Brazilian Industrial Group. R\&D Management. Cambridge - UK 2016.

MINTZBERG, H., AHLSTRAND, B., LAMPEL, J. Safari de estratégia: um roteiro pela selva do planejamento estratégico. Porto Alegre: Bookman, 2000.

MOTTA, P.R. Transformação organizacional. Rio de Janeiro: Qualitymark, 1998.

NELSON, R.R.; WINTER, S.G. An Evolutionary Theory os Economic Change. Cambridge, Mass; London: : The Belknap Press of of Havard University Press, 1982.

O'CONNOR, G.C. Innovation: From Process to Function. Journal of Product Innovation Management, v. 29, n. 3, p. 361-363, 2012. https://doi.org/10.1111/j.1540-5885.2012.00909.x

O'CONNOR, G.C. et al. Grabbing Lightning: Building a Capability for Breakthrough Innovation. San Francisco: John Wiley \& Sons, 2008. 332p.

SKARZYNSKI, P.; GIBSON, R. Inovação: prioridade número 1 - o caminho para transformações nas organizações. Campus, 2008. 336p.

TEECE, D.J. Explicating dynamic capabilities: The nature and microfoundations of (sustainable) enterprise performance. Strategic Management Journal, v. 28, n. 13, p. 13191350, Dec 2007. https://doi.org/10.1002/smj.640

WOOD JÚNIOR, T. Mudança organizacional. 4 ed. São Paulo: Atlas, 2004. 334p.

YIN, R.K. Case study research: design and methods. 3ed..Thousand Oaks, CA: Sage, 2004.

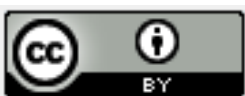

Artigo recebido em 03/03/2016 e aceito para publicação em 29/04/2017 DOI: http://dx.doi.org/10.14488/1676-1901.v17i2.2376 
ADENDO I - CRONOLOGIA DOS EVENTOS PARA O PESQUISADOR-AUTOR

Quadro 3 - Cronologia dos Eventos

\begin{tabular}{|c|c|c|c|}
\hline ORDEM & DESCRIÇÃO & ANO & MÊS \\
\hline 1 & $\begin{array}{l}\text { Contratação do Pesquisador-Autor para assumir o } \\
\text { "Núcleo de Gestão da Inovação" }\end{array}$ & 2010 & 10 \\
\hline 2 & $\begin{array}{l}\text { Reuniões com agentes internos envolvidos com } \\
\text { inovação }\end{array}$ & 2010 & 10 \\
\hline 3 & Transferência de atribuições do Agente-3 para Agente-6 & 2010 & 10 \\
\hline 4 & $\begin{array}{l}\text { Pesquisador-Autor conhece tentativa frustrada de } \\
\text { geração anterior ao P\&D-25 junto ao FINEP }\end{array}$ & 2010 & 10 \\
\hline 5 & Apresentação do "LEI DO BEM" em evento na UFMG & 2010 & 11 \\
\hline 6 & $\begin{array}{l}\text { Início da interação com setor de auditoria de projetos de } \\
\text { inovação da CEMIG }\end{array}$ & 2010 & 11 \\
\hline 7 & Estudo de viabilidade do P\&D-3 & 2010 & 12 \\
\hline 8 & $\begin{array}{c}\text { Reestruturação do Grupo por princípios de Governança } \\
\text { Coorporativa }\end{array}$ & 2011 & 1 \\
\hline 9 & Ideia do P\&D-7 & 2011 & 2 \\
\hline 10 & $\begin{array}{l}\text { Parceria com CEFET-MG para desenvolvimento do } \\
\text { P\&D-4 em chamada do "P\&D-ANEEL" da CEMIG }\end{array}$ & 2011 & 3 \\
\hline 11 & $\begin{array}{c}\text { Implementação de sistema de farol para controle de } \\
\text { avanço de projetos de inovação }\end{array}$ & 2011 & 3 \\
\hline 12 & Identificação do "EDITAL SENAI-SESI DE INOVAÇÃO” & 2011 & 3 \\
\hline 13 & Aprovação interna do P\&D-5 & 2011 & 3 \\
\hline 14 & $\begin{array}{c}\text { Reunião de alinhamento do P\&D-5 com setor de } \\
\text { "Engenharia do Produto" }\end{array}$ & 2011 & 3 \\
\hline 15 & $\begin{array}{l}\text { Apresentação do conceito dos P\&D-5 e P\&D-6 à } \\
\text { coordenação do "EDITAL SENAI-SESI DE INOVAÇÃO" }\end{array}$ & 2011 & 3 \\
\hline 16 & Tentativa de parceria com SENAI CETEL & 2011 & 3 \\
\hline 17 & $\begin{array}{c}\text { Problemas com movimentação de contas bancárias de } \\
\text { projetos junto ao Departamento Financeiro }\end{array}$ & 2011 & 5 \\
\hline 18 & $\begin{array}{c}\text { Parceria com SENAI EUVALDO LODI para } \\
\text { desenvolvimento do P\&D-5 em chamada do "EDITAL } \\
\text { SENAI-SESI DE INOVAÇÃO" }\end{array}$ & 2011 & 5 \\
\hline 19 & $\begin{array}{c}\text { Apresentação de conjunto de melhorias internas por } \\
\text { grupo de colaboradores }\end{array}$ & 2011 & 5 \\
\hline 20 & Enquadramento do P\&D-3 no “LEI DO BEM” & 2011 & 5 \\
\hline 21 & $\begin{array}{c}\text { Submissão do P\&D-5 ao “EDITAL SENAI-SESI DE } \\
\text { INOVAÇÃO” }\end{array}$ & 2011 & 5 \\
\hline 22 & $\begin{array}{c}\text { Início antecipada do P\&D-5 em conjunto com SENAI } \\
\text { EUVALDO LODI }\end{array}$ & 2011 & 6 \\
\hline 23 & $\begin{array}{c}\text { Parceria com CEFET-MG e microempresa para } \\
\text { submissão do P\&D-7 em chamada do "EDITAL CEMIG- } \\
\text { FAPEMIG" }\end{array}$ & 2011 & 7 \\
\hline 24 & $\begin{array}{l}\text { Aprovação do P\&D-5 no “EDITAL SENAI-SESI DE } \\
\text { INOVAÇÃO" }\end{array}$ & 2011 & 7 \\
\hline 25 & Convite à EP-6 para participação em licitações & 2011 & 8 \\
\hline 26 & $\begin{array}{l}\text { Parceria com CEFET-MG para desenvolvimento do } \\
\text { P\&D-8 em chamada do "EDITAL CEMIG-FAPEMIG" }\end{array}$ & 2011 & 8 \\
\hline 27 & $\begin{array}{c}\text { Submissão do P\&D-7 e P\&D-8 ao "EDITAL CEMIG- } \\
\text { FAPEMIG" }\end{array}$ & 2011 & 8 \\
\hline 28 & Efetivação do Pesquisador-Autor & 2011 & 8 \\
\hline 29 & Visita à EP-2 e identificação do P\&D-9 & 2011 & 9 \\
\hline 30 & $\begin{array}{c}\text { Parceria com IEL no "PROGRAMA DE GERAÇÃO DE } \\
\text { IDEIAS" }\end{array}$ & 2011 & 9 \\
\hline 31 & Implantação de "Comitê de P,D\&l" & 2011 & 10 \\
\hline 32 & Interação do NGI com Planejamento Estratégico & 2011 & 10 \\
\hline 33 & $\begin{array}{c}\text { Apresentação do programa “PRÓ-INOVAÇÃO” em } \\
\text { evento na FIEMG }\end{array}$ & 2011 & 10 \\
\hline 34 & Desafio em compra de materiais para P\&D-5 & 2011 & 10 \\
\hline 35 & Nova coordenação de Planejamento Estratégico & 2011 & 11 \\
\hline 36 & Aprovação de fomento junto ao BDMG para P\&D-2, & 2011 & 11 \\
\hline
\end{tabular}




\begin{tabular}{|c|c|c|c|}
\hline & P\&D-5 e P\&D-10 & & \\
\hline 37 & $\begin{array}{c}\text { Tentativa frustrada de adequação de sistema de gestão } \\
\text { coorporativo para projetos de inovação }\end{array}$ & 2011 & 11 \\
\hline 38 & Contratação da AGENTE-19 & 2012 & 1 \\
\hline 39 & Ajustes internos para atendimento ao "LEI DO BEM" & 2012 & 1 \\
\hline 40 & Submissão do P\&D-9 ao FINEP e BNDES & 2012 & 1 \\
\hline 41 & $\begin{array}{c}\text { Aprovação de P\&D-7 e P\&D-8 no "EDITAL CEMIG- } \\
\text { FAPEMIG" }\end{array}$ & 2012 & 1 \\
\hline 42 & $\begin{array}{c}\text { Validação de relatório do “LEI DO BEM" junto à } \\
\text { empresas e consultoria }\end{array}$ & 2012 & 3 \\
\hline 43 & $\begin{array}{c}\text { Inclusão do NGI em grupo de discussão do "LEI DO } \\
\text { BEM" }\end{array}$ & 2012 & 3 \\
\hline 44 & Rompimento com consultoria da "LEI DO BEM" & 2012 & 3 \\
\hline 45 & $\begin{array}{l}\text { Reprovação de fomento junto ao BDMG para P\&D-6 e } \\
\text { P\&D-13 }\end{array}$ & 2012 & 3 \\
\hline 46 & $\begin{array}{c}\text { Proposta de suporte ao "LEI DO BEM" por outra } \\
\text { consultoria especialista }\end{array}$ & 2012 & 3 \\
\hline 47 & Prorrogação de prazo do P\&D-2 & 2012 & 4 \\
\hline 48 & Aprovação de fomento junto ao FINEP para P\&D-9 & 2012 & 4 \\
\hline 49 & $\begin{array}{l}\text { Adequação de sistema de gestão coorporativo para } \\
\text { projetos de inovação }\end{array}$ & 2012 & 4 \\
\hline 50 & Alteração do P\&D-7 para o P\&D-11 & 2012 & 5 \\
\hline 51 & Declínio do P\&D-11 pela CEMIG & 2012 & 5 \\
\hline 52 & $\begin{array}{l}\text { Priorização do P\&D-14 na EP-3 para submissão ao } \\
\text { "EDITAL SENAI-SESI DE INOVAÇÃO" }\end{array}$ & 2012 & 5 \\
\hline 53 & $\begin{array}{c}\text { Identificação do P\&D-15 e P\&D-16 em reunião de } \\
\text { Comitê de P,D\&I }\end{array}$ & 2012 & 5 \\
\hline 54 & Críticas internas ao P\&D-3 & 2012 & 5 \\
\hline 55 & Alteração do P\&D-8 para o P\&D-12 & 2012 & 5 \\
\hline 56 & Definição da gestão do P\&D-3 & 2012 & 5 \\
\hline 57 & $\begin{array}{c}\text { Submissão do P\&D-11 P\&D-12 e P\&D-19 à } \\
\text { ELETROBRÁS }\end{array}$ & 2012 & 5 \\
\hline 58 & Reunião para discussão de viabilidade do P\&D-17 & 2012 & 5 \\
\hline 59 & $\begin{array}{l}\text { Problemas com apontamento de horas de } \\
\text { colaboradores de fábrica em projetos de inovação }\end{array}$ & 2012 & 5 \\
\hline 60 & $\begin{array}{c}\text { Submissão do P\&D-14 e P\&D-17 ao "EDITAL SENAI- } \\
\text { SESI DE INOVAÇÃO" }\end{array}$ & 2012 & 5 \\
\hline 61 & Ampliação da dedicação do AGENTE-6 & 2012 & 6 \\
\hline 62 & $\begin{array}{c}\text { Priorização do P\&D-23 dentre as demandas da CEB } \\
\text { DISTRIBUIÇÃO }\end{array}$ & 2012 & 6 \\
\hline 63 & Elaboração de proposta de financiamento para P\&D-21 & 2012 & 6 \\
\hline 64 & Ideia do P\&D-22 & 2012 & 7 \\
\hline 65 & Falhas na gestão à distância do P\&D-9 & 2012 & 7 \\
\hline 66 & $\begin{array}{l}\text { Desenvolvimento de relatório específico para atender ao } \\
\text { "LEI DO BEM" }\end{array}$ & 2012 & 8 \\
\hline 67 & Convite da FITEC para participação em P\&D-20 & 2012 & 8 \\
\hline 68 & Visita à EP-2 com agente da FINEP & 2012 & 9 \\
\hline 69 & Reunião de apresentação do “INOVA PETRO” & 2012 & 9 \\
\hline 70 & $\begin{array}{c}\text { Reprovação do P\&D-14 e P\&D-17 no "EDITAL SENAI- } \\
\text { SESI DE INOVAÇÃO" }\end{array}$ & 2012 & 9 \\
\hline 71 & $\begin{array}{c}\text { Interação com equipe do "PROGRAMA DE GERAÇÃO } \\
\text { DE IDEIAS" }\end{array}$ & 2012 & 10 \\
\hline 72 & $\begin{array}{l}\text { Parceria com CEFET-MG para desenvolvimento do } \\
\text { P\&D-12 em chamada do "EDITAL CEMIG-FAPEMIG" }\end{array}$ & 2012 & 10 \\
\hline 73 & Submissão do P\&D-30 ao "EDITAL CEMIG-FAPEMIG" & 2012 & 10 \\
\hline 74 & $\begin{array}{l}\text { Problemas com contratação de transporte e testes do } \\
\text { P\&D-5 }\end{array}$ & 2012 & 10 \\
\hline 75 & $\begin{array}{l}\text { Submissão do P\&D-24, 25, 26, 27, } 28 \text { e } 29 \text { ao "INOVA } \\
\text { PETRO" }\end{array}$ & 2012 & 10 \\
\hline 76 & Início de relacionamento entre EP-6 e grande empresa & 2012 & 10 \\
\hline 77 & $\begin{array}{l}\text { Submissão do P\&D-31 e P\&D-32 ao BDMG com } \\
\text { aprovação de financiamento para o último }\end{array}$ & 2012 & 11 \\
\hline
\end{tabular}




\begin{tabular}{|c|c|c|c|}
\hline 78 & Finalização do P\&D-2 junto à CEMIG & 2012 & 12 \\
\hline 79 & Concorrência do PETROBRAS envolvendo o P\&D-27 & 2012 & 12 \\
\hline 80 & $\begin{array}{c}\text { Apresentação de projetos do "INOVA PETRO" a } \\
\text { membro do Conselho de Administração }\end{array}$ & 2012 & 12 \\
\hline 81 & Início do P\&D-12 & 2012 & 12 \\
\hline 82 & $\begin{array}{l}\text { Aval formal da CEMIG para execução de novo } \\
\text { orçamento do P\&D-12 }\end{array}$ & 2013 & 1 \\
\hline 83 & Efetivação da AGENTE-19 & 2013 & 1 \\
\hline 84 & Aprovação de proposta inicial ao “INOVA PETRO" & 2013 & 1 \\
\hline 85 & Convite da UERJ para participação em P\&D-40 & 2013 & 1 \\
\hline 86 & Venda da EP-3 & 2013 & 2 \\
\hline 87 & Pré-aprovação do P\&D-23 & 2013 & 3 \\
\hline 88 & $\begin{array}{c}\text { Submissão do P\&D-11 P\&D-12 e P\&D-19 à } \\
\text { TRACTEBEL ENERGIA }\end{array}$ & 2013 & 3 \\
\hline 89 & Ideia do P\&D-35 & 2013 & 4 \\
\hline 90 & $\begin{array}{l}\text { Oportunidades comerciais identificadas associadas ao } \\
\text { P\&D-29 }\end{array}$ & 2013 & 4 \\
\hline 91 & $\begin{array}{c}\text { Parceria com UNIFEI e microempresa para submissão } \\
\text { do P\&D-36 ao "INOVA ENERGIA" }\end{array}$ & 2013 & 4 \\
\hline 92 & $\begin{array}{c}\text { Convite ao NGI para participação em grupos de } \\
\text { Planejamento Estratégico }\end{array}$ & 2013 & 4 \\
\hline 93 & Elaboração de proposta de financiamento para P\&D-41 & 2013 & 4 \\
\hline 94 & $\begin{array}{c}\text { Submissão do P\&D-15, 22, 36, 37, 38, } 39 \text { ao "INOVA } \\
\text { ENERGIA" }\end{array}$ & 2013 & 4 \\
\hline 95 & $\begin{array}{l}\text { Contratação de consultoria para valoração de cotas da } \\
\qquad \text { EP-6 }\end{array}$ & 2013 & 4 \\
\hline 96 & Testes de protótipo do P\&D-5 no exterior & 2013 & 5 \\
\hline 97 & $\begin{array}{l}\text { Nova concorrência do PETROBRAS envolvendo o P\&D- } \\
27\end{array}$ & 2013 & 5 \\
\hline 98 & Proposta de assessoria à EP-6 & 2013 & 5 \\
\hline 99 & Início do contrato do P\&D-9 com FINEP & 2013 & 5 \\
\hline 100 & $\begin{array}{l}\text { Questionamentos da CEMIG em relação à gestão do } \\
\text { P\&D-12 }\end{array}$ & 2013 & 6 \\
\hline 101 & Convite da UFMG para participação em P\&D-42 & 2013 & 6 \\
\hline 102 & Ideia do P\&D-44 a partir do P\&D-42 & 2013 & 6 \\
\hline 103 & Convite da UFMG para participação em P\&D-43 & 2013 & 6 \\
\hline 104 & Negociação frustrada entre EP-6 e grande empresa & 2013 & 6 \\
\hline 105 & Submissão do P\&D-2, 5, 9, 10, 33 ao "LEI DO BEM" & 2013 & 6 \\
\hline 106 & $\begin{array}{l}\text { Apresentação de projetos do "INOVA PETRO" ao setor } \\
\text { de equity da FINEP }\end{array}$ & 2013 & 7 \\
\hline 107 & Declínio de propostas pela ELETROBRÁS & 2013 & 7 \\
\hline 108 & $\begin{array}{l}\text { Desalinhamento de projetos em segunda etapa do } \\
\text { "INOVA PETRO" }\end{array}$ & 2013 & 8 \\
\hline 109 & Ideia do P\&D-45 declinada pelo volume de atividades & 2013 & 8 \\
\hline 110 & Tensões entre Pesquisador-Autor e alta direção & 2013 & 8 \\
\hline 111 & Desligamento do Pesquisador-Autor & 2013 & 8 \\
\hline 112 & Reestruturação e criação de "Departamento de P\&D" & 2013 & 8 \\
\hline
\end{tabular}

